\title{
Technology of Rapid Prototyping SMD MOX Gas Sensors.
}

\author{
Nikolay N. Samotaev ${ }^{1}$, Konstantin Yu. Oblov $^{1}$, Anastasia V. Ivanova ${ }^{1}$ \\ ${ }^{1}$ National Research Nuclear University MEPHI, 115409, Kasirskoe highway 31, Moscow, Russian \\ nnsamotaev@mephi.ru
}

\begin{abstract}
The perspective laser micromilling technology for ceramic MEMS producing of microhotplate in the surface mounted device (SMD) package for the metal oxide (MOX) sensor is describing. There are discusses technological and economic aspects of small-scale production of gas MOX sensors. The main technical factors affecting the use of sensors in various applications are presented.
\end{abstract}

Key words: SMD packaging, MOX gas sensor, Ceramic, MEMS.

\section{Introduction}

The modern MOX sensor is a combination of several factors - a microhotplate (responsible for power consumption and temperature operation mode), MOX gas sensitive layer (responsible for sensitivity to target gases) and a package (responsible for the possible application in which the sensor is used). Last decades MOX gas sensors development there is a concentration of efforts, in the field of technologies allowing obtaining a cheap product for mass applications in the manner of indoor air quality (IAQ) monitoring. Similar solutions were found by the leading manufacturers of MOX sensors (Figaro, Sensirion, Bosch, AMS, SGX and etc.) [1-5]. Technological solutions that make it possible to obtain a sensor with satisfactory characteristics for such application consist in the use of a combination of silicon MEMS microhotplate [15] and plastic [1-2], metal-plastic [3] or metalceramic $[4,5]$ SMD package. But what to do if for scientific groups which, at the moment there is no market or is it too small for the return of investment in silicon MEMS technology and the production of specialized plastic or ceramic SMD package? In that case need technology process close to $3 \mathrm{D}$ prototype philosophy rapid, simple and cheap with ratio a single sample to total production cost.

\section{Experiment}

As a technology for manufacturing ceramic MEMS microhotplate and SMD packages, it is proposed to use laser micromilling of monolithic $\mathrm{Al}_{2} \mathrm{O}_{3}$ and screen-print, jet (aerosol) printing technologies to form platinum metallization on MEMS and package. The equipment involved in the described technology does not need clean rooms and is widely represented on the market. The software for technology is simple and accessible at the student level. The only specialized product of the proposed technology is the adaptation of machine vision to obtain the minimum possible size of the MEMS structure of the microheater and the deposition of the MOX gas sensitive layer and platinum metallization, as well as the translation program of the 3D models of MEMS microhotplate and 2-D topology of metallization to a 4-axis laser micromilling facilities for automatic production. Technological steps are follows:

3D modeling of MEMS microheater, top and bottom part of the package (Fig. 1a, b) in *.stl format (Autodesk Inventor or SolidWorks program) and 2D modeling topology of MEMS metallization in *.dxf format (Autodesk AutoCAD program).

Simulation of the MEMS microheater parameters in the ANSYS program for prediction approximate representations about the MOX sensor thermal characteristics (optional)

Laser micromilling of monolithic ceramics on a 4-axis laser facility by using 3D model of MEMS microheater, top and bottom part of the package (Fig 2).

Deposition of platinum metallization using 2D model topology and technical annealing of metallization on MEMS according 
to specification on screen-print, jet or aerosol platinum materials (Fig. 1c).

- Laser micro milling of metallization according 2D model (optional)

- Deposition and annealing of the MOX gas sensitive layer on the MEMS microhotplate

- Assembling and conglutination by special glass single parts of sensor into a monolith package, like schematically represented for SOT-23 package on Fig. 1d.

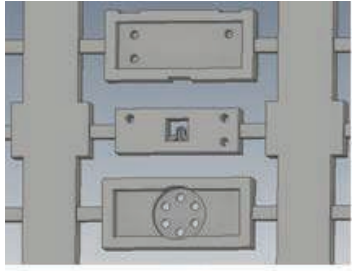

a

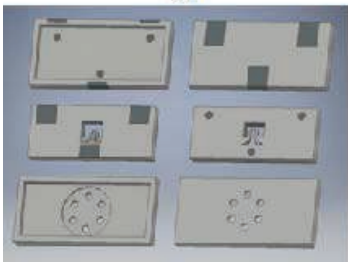

C

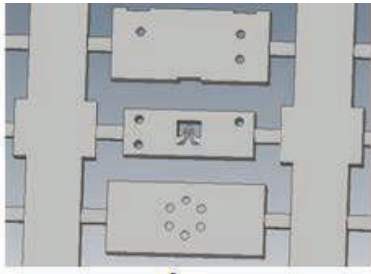

b

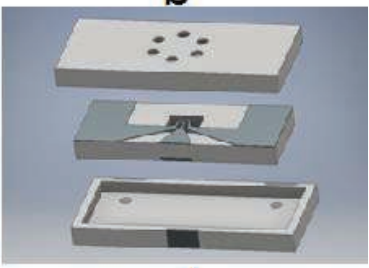

d
Fig. 1. a) Bottom and b) Top view of $3 D$ model of microhotplate and parts of SMD package as a task for laser micromilling. c) Scheme of metallization topology in 3D model. d) Scheme of assembling MEMS microhotplate in SMD package.

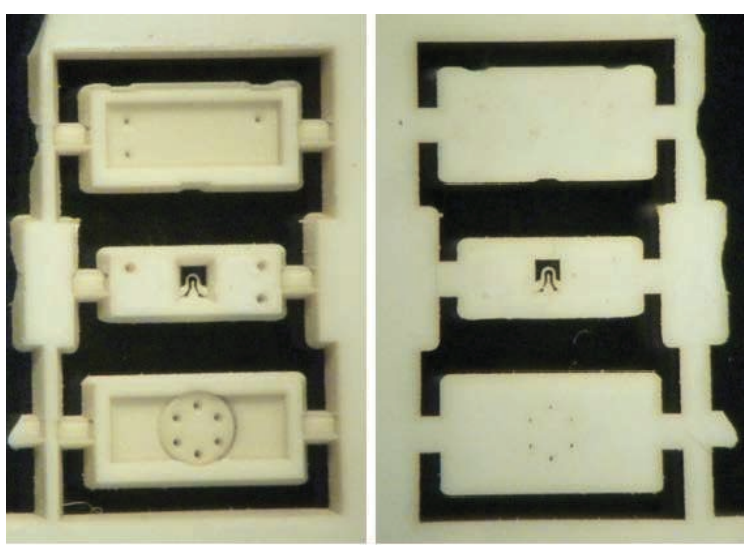

Fig. 2. Photos of bottom (left) and top (right) views on $250 \mu \mathrm{m}$ thick Al2O3 substrate with part of SMD MOX gas sensor after laser micromilling by 4-x axis CNC 1,064 um fiber laser tool.

Using present approach, experiments were carried out to fabricate a MEMS microhotplate, whose track width was $30 \mu \mathrm{m}$ and a thickness of $20 \mu \mathrm{m}$ (see Fig.3). The power consumption of MEMS microhotplate at $450{ }^{\circ} \mathrm{C}$ was approximately $250 \mathrm{~mW}\left(350 \mathrm{~mW}\right.$ at $\approx 600{ }^{\circ} \mathrm{C}$ "burning" platinum temperature, see Fig.3d). Current results gives the prospect that manufacture MOX sensor in the SMD SOT-23 package type (max dissipating power by formfactor of package is $350 \mathrm{~mW}$ [6]) for surface mounting in a tape is available by using described technology.

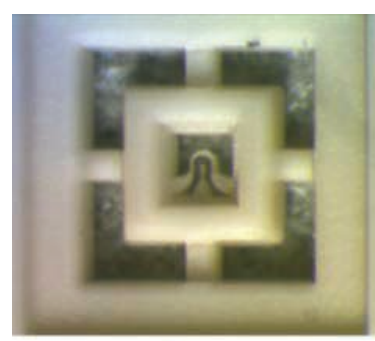

a

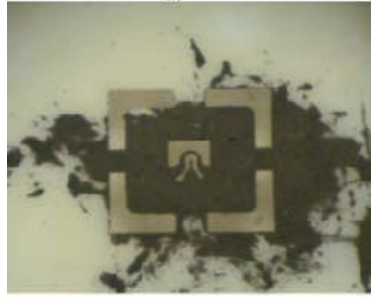

C

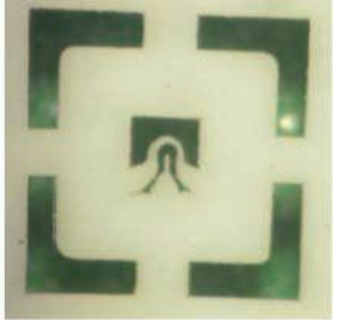

b

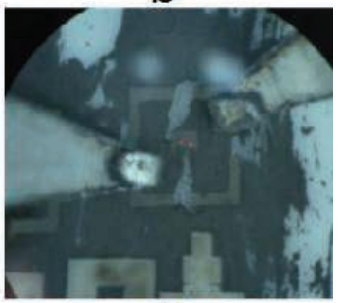

d
Fig. 3. a) Bottom view of MEMS after laser micro milling. Chip size $1 \times 1 \mathrm{~mm}$ by border of internal frame. b) Top view of MEMS. Internal window size 0,3x0,3 $\mathrm{mm}$. Central track width is $30 \mu \mathrm{m}$. c) Microheater track width 30 micron under cower Pt paste. d) Testing MEMS microhotplate under voltage load (pins). Hot area at $\approx 600^{\circ} \mathrm{C}$

\section{Conclusion}

Experiments with laser micromilling of monolithic $\mathrm{Al}_{2} \mathrm{O}_{3}$ ceramics confirmed possibility to produce MEMS microhotplate for MOX gas sensor in SMD package in the form-factor SOT23. Developed technology process are close to 3D prototype philosophy - rapid, simple and cheap.

\section{Acknowledgement}

This work was supported by the Ministry Education and Science Russian Federation (Grant No. 14.584.21.0030 from 22.11.2017, unique identifier RFMEFI58417X0030) in frame of joint Russian-Korean project.

\section{References}

[1] https://www.sensirion.com/en/environmentalsensors/gas-sensors/

[2] http://ams.com/eng/Products/EnvironmentalSensors/Gas-Sensors/AS-MLV-P2

[3] https://www.boschsensortec.com/bst/products/all_products/bme680

[4] https://www.sgxsensortech.com/content/uploads/ 2014/08/1107_Datasheet-MiCS-2714.pdf

[5] http://www.figarosensor.com/products/entry/tgs81 00.html

[6] https://www.diodes.com/assets/Datasheets/ds18 001.pdf 